\title{
Lumped Parameter Estimation of a Stochastic Process of Second Order Using the Second Moment and Recursiveness
}

\author{
Romeo Urbieta Parrazales ${ }^{1}$, José de Jesús Medel Juárez ${ }^{1}$, Karen Alicia Aguilar Cruz ${ }^{1}$, \\ Rosaura Palma Orozco ${ }^{2}$ \\ ${ }^{1}$ Centro de Investigación en Computación - Instituto Politécnico Nacional, CDMX, \\ Mexico \\ ${ }^{2}$ Escuela Superior de Cómputo - Instituto Politécnico Nacional, CDMX, \\ Mexico \\ rurbieta700@gmail.com,jjmedelj@yahoo.com.mx, karen_ali320@hotmail.com, \\ rpalma@ipn.mx
}

\begin{abstract}
In this article, a stochastic algorithm is briefly presented based on the one of second moment applied to a stochastic process model of second order. The design initially consisted in formulating the state equation model and the stochastic outputs, in order to apply the second moment using the internal product of Martingale and the stochastic operators of the expectation, variance and covariance. The design results generated the formulas on: the covariances and the internal product variances to calculate the lumped estimation parameters, the error functional based on the mean quadratic error, the output variable as a function of the estimation parameters obtained. Furthermore, the recursive form was formulated in this design starting from the premise of the obtained results using the second stochastic moment. The main interest lays on the recursive form, because this is the one capable of being implemented in a digital system. In order to observe the precision and the convergence of the estimation parameters and the output variables, Matlab-based figures are shown.
\end{abstract}

Keywords: Linear stochastic systems, parameter estimation, second moment.

\section{Introduction}

The random input and output variables of a noisy process can be modeled by means of experimental data obtained from measurements carried out during time intervals $\mathrm{T}$. These models are called Black Box Models (Fig. 1) [1, 2].

The Black Box Models are called this way, because their internal states are not known. One method to determine these unknown states starting from observable states are the so called methods of identified states based on the mean quadratic error [3]. 


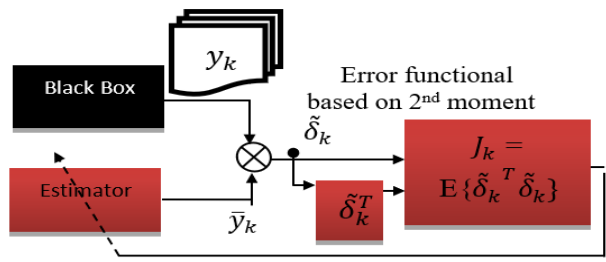

Fig. 1. Stochastic Estimation based on the second moment.

The method relies on turning the unknown or non-measurable states $x_{k}$ into an identified state $\hat{x}_{k}$ by means of the stochastic conditions of the observable state $y_{k}$, based on the identified error (1):

$$
\tilde{\delta}_{k}=y_{k}-\bar{y}_{k}
$$

The error can lead to different methods of stochastic estimation and is hereby known as the innovation process.

The error functional from the identification error $\tilde{\delta}_{k}$ using the second moment of probability, is defined as the expected value of the Euclidean norm of $\tilde{\delta}_{k}[4,5,6]$ :

$$
J_{k}\left(\tilde{\delta}_{k}\right)=\mathrm{E}\left\{\tilde{\delta}_{k}{ }^{T} \tilde{\delta}_{k}\right\}=\left(y_{k}-\bar{y}_{k}\right)^{T}\left(y_{k}-\bar{y}_{k}\right)
$$

On the other hand, within the linear stochastic model, the observable state was represented by means of finite differences equations of $2^{\text {nd }}$ order (3):

$$
\frac{d^{2} f(y)}{d y^{2}} \cong(f(y)-2 f(y(k-1))+f(y(k-2)) .
$$

As it can be observed in (1) a system of this type has two delays and a negative sign in one of its parameters $[7,8,9,10,11]$.

As we mentioned before, based on a second order state and a quadratic mean of second moment, the main purposes of this work are to design the estimation state based on this second moment, further on, to identify the stochastic output variable and finally, to determine the precision by means of the error functional. Additionally, the parameters and the variables of interest will be plotted.

This article was structured in the following manner: abstract, second moment, stochastic recursive algorithm, quadratic mean error functional, experiment, conclusions and bibliography.

\section{Simplified Stochastic Output Model.}

Like it was said in this introduction, a stochastic system based on noisy output and input measurements taken during a certain number of time intervals $\mathrm{T}$, can adopt the following linear model in differences like the state equation (4) with stochastic output (5) $[9,10]$ : 


$$
\begin{gathered}
x_{k+1}=a_{1} x_{k}+a_{2} x_{k-1}+b w_{k}, \\
y_{k}=c x_{k}+d v_{k} .
\end{gathered}
$$

Delaying (4) and (5) we have (6) and (7) respectively

$$
\begin{gathered}
x_{k}=a_{1} x_{k-1}+a_{2} x_{k-2}+b w_{k-1}, \\
y_{k-1}=c x_{k-1}+d v_{k-1} .
\end{gathered}
$$

Now, solving for the delayed state from (7) yields (8), and delaying (8) gives (9):

$$
\begin{aligned}
& x_{k-1}=c^{-1} y_{k-1}-d c^{-1} v_{k-1}, \\
& x_{k-2}=c^{-1} y_{k-2}-d c^{-1} v_{k-2} .
\end{aligned}
$$
(11):

Substituting (8) and (9) in (6) gives (10). Besides, substituting (10) in (5) yields

$$
\begin{gathered}
x_{k}=a_{1} c^{-1} y_{k-1}+a_{2} c^{-1} y_{k-2}-a_{1} d c^{-1} v_{k-1}-a_{2} d c^{-1} v_{k-2}+b w_{k-1}, \\
y_{k}=a_{1} y_{k-1}+a_{2} y_{k-2}-a_{1} d v_{k-1}-a_{2} d v_{k-2}+b c w_{k-1}+d v_{k} .
\end{gathered}
$$

And from (11) the noise is (12) leading to the simplified stochastic output (13):

$$
\begin{gathered}
V_{k}=-a_{1} d v_{k-1}-a_{2} d v_{k-2}+b c w_{k-1}+d v_{k}, \\
\bar{y}_{k}=a_{1} y_{k-1}+a_{2} y_{k-2}+V_{k} .
\end{gathered}
$$

\section{Stochastic Algorithm Based on the Second Moment}

The second moment is a technique used in probability theory to demonstrate that a random variable (RV) has a positive probability. Generally, the method consists in delimiting the probability that a RV fluctuates away from its mean. The method involves a comparison of the second moment of the RV and the square of the first moment. Hence: $\mu_{2}=E\left((Y-\mu)^{2}\right)$, where $\mathrm{Y}$ is the RV and $\mu$ is the mean [12].

From (13), we can obtain (14), that is (15), where $\bar{a}=\left[\begin{array}{ll}a_{1} & a_{2}\end{array}\right]$, and $\bar{z}_{k-1}=$ $\left[y_{k-1}: y_{k-2}\right]^{T}$ :

$$
\begin{gathered}
y_{k}=\bar{a} \bar{z}_{k-1}+V_{k}, \\
y_{k}=\left[\begin{array}{ll}
a_{1} & a_{2}
\end{array}\right]\left[\begin{array}{ll}
y_{k-1} & y_{k-2}
\end{array}\right]^{T}+V_{k},
\end{gathered}
$$

Taking the second moment of probability from (14) for the variable $z_{k-1}^{T}[13,14]$, we have (16), that once solved gives (17):

$$
\begin{aligned}
& E\left\{y_{k} \bar{z}_{k-1}^{T}\right\}=\bar{a} E\left\{\bar{z}_{k-1} \bar{z}_{k-1}^{T}\right\}+E\left\{V_{k} \bar{z}_{k-1}^{T}\right\}, \\
& E\left\{y_{k} \bar{z}_{k-1}^{T}\right\}-E\left\{V_{k} \bar{z}_{k-1}^{T}\right\}=\bar{a} E\left\{\bar{z}_{k-1} \bar{z}_{k-1}^{T}\right\} .
\end{aligned}
$$

Here is the estimated parameter based on the second moment (18): 
Romeo Urbieta Parrazales, José de Jesús Medel Juárez, Karen Alicia Aguilar Cruz, et al.

$$
\bar{a}_{k}=\left(E\left\{y_{k} \bar{z}_{k-1}^{T}\right\}-E\left\{V_{k} \bar{z}_{k-1}^{T}\right\}\right)\left(E\left\{\bar{z}_{k-1} \bar{z}_{k-1}^{T}\right\}\right)^{+} .
$$

From (18), the stochastic estimated parameter is defined as a direct function to the covariance $p_{k}$, and inverse to the covariance $q_{k}$ as indicated in (19):

$$
\bar{a}_{k} \triangleq \frac{p_{k}}{q_{k}}
$$

where, $\bar{a}_{k}, p_{k}$, and $q_{k}$ are vectors of $1 \times 2,1 \times 2$ and $2 \times 2$ dimensions, respectively.

To calculate the covariances $\mathrm{p}_{\mathrm{k}}$ and $\mathrm{q}_{\mathrm{k}}$, the stochastic covariance is represented by the expectation of the internal products of the output variable, which is convoluted with the same output, but now with a delay, (20) [11]:

$$
\left[\begin{array}{ll}
\bar{p}_{1, k} & \bar{p}_{2, k}
\end{array}\right]=E\left(\left\{y_{k} \bar{z}_{k-1}^{T}\right\}-\left\{V_{k} \bar{z}_{k-1}^{T}\right\}\right) .
$$

The stochastic expectation comes represented as a sequence from zero to $\mathrm{k}$ samples and is expressed by means of a sum [15] as it can be seen in (21):

$$
\left[\begin{array}{ll}
\bar{p}_{1, k} & \bar{p}_{2, k}
\end{array}\right]=\frac{1}{k}\left[\sum_{i=0}^{k}\left(\left[\begin{array}{ll}
y_{i} y_{i-1} & y_{i} y_{i-2}
\end{array}\right]-\left[\begin{array}{ll}
V_{i} y_{i-1} & V_{i} y_{i-2}
\end{array}\right]\right)\right]
$$

Solving the sums from 0 to $\mathrm{k}$ samples, the two vectors of the covariances [13] $p_{1, k}$ and $p_{2, k}$, are obtained as in (22):

$$
\begin{aligned}
& {\left[\bar{p}_{1, k} \quad \bar{p}_{2, k}\right]=} \\
& \frac{1}{k^{2}}\left[y_{k} y_{k-1}-V_{k} y_{k-1}+(k-1)^{2} \bar{p}_{1, k-1} \quad y_{k} y_{k-2}-V_{k} y_{k-2}+(k-1)^{2} \bar{p}_{2, k-1}\right] \text {. }
\end{aligned}
$$

While the covariance $q_{k}$ comes expressed as a quadratic matrix of $2 \times 2(23)$, in this case, the matrix turns out to be singular yielding into a pseudoinverse matrix.

$$
\begin{gathered}
{\left[\begin{array}{ll}
\bar{q}_{11, k} & \bar{q}_{12, k} \\
\bar{q}_{21, k} & \bar{q}_{22, k}
\end{array}\right]=E\left\{\bar{z}_{k-1} \bar{z}_{k-1}^{T}\right\}} \\
=E\left\{\left[\begin{array}{l}
y_{k-1} \\
y_{k-2}
\end{array}\right]\left[\begin{array}{ll}
y_{k-1} & y_{k-2}
\end{array}\right]\right\}=E\left[\begin{array}{lr}
y_{k-1}^{2} & y_{k-1} y_{k-2} \\
y_{k-2} y_{k-1} & y_{k-2}^{2}
\end{array}\right] .
\end{gathered}
$$

Solving for the expectation operator over the internal product, the covariances matrix $q_{k}$ is obtained in (24):

$$
\left[\begin{array}{ll}
q_{11, k} & q_{12, k} \\
q_{21, k} & q_{22, k}
\end{array}\right]=\frac{1}{k^{2}} \sum_{i=0}^{k}\left[\begin{array}{lr}
y_{i-1}^{2} & y_{i-1} y_{i-2} \\
y_{i-2} y_{i-1} & y_{i-2}^{2}
\end{array}\right]
$$

Now, by inserting the sums into each one of the terms within the pseudoinverse matrix, the result is (25).

Developing the samples in each one of the sums from 0 to $\mathrm{k}$ samples leads to obtain the sequential form of each one of the components of the pseudoinverse matrix (26) of the covariance $q_{k}$. 


$$
\begin{gathered}
{\left[\begin{array}{cc}
\bar{q}_{11, k} & \bar{q}_{12, k} \\
\bar{q}_{21, k} & \bar{q}_{22, k}
\end{array}\right]=\frac{1}{k^{2}}\left(\left[\begin{array}{ll}
\sum_{i=0}^{k} y_{i-1}^{2} & \sum_{i=0}^{k} y_{i-2} y_{i-1} \\
\sum_{i=0}^{k} y_{i-1} y_{i-2} & \sum_{i=0}^{k} y_{i-2}^{2}
\end{array}\right]\right),} \\
{\left[\begin{array}{ll}
\bar{q}_{11, k} & \bar{q}_{12, k} \\
\bar{q}_{21, k} & \bar{q}_{22, k}
\end{array}\right]=\frac{1}{k^{2}}\left(\left[\begin{array}{cc}
q_{11, k} & q_{12, k} \\
q_{21, k} & q_{22, k}
\end{array}\right]\right),}
\end{gathered}
$$

where the components of the pseudoinverse matrix are (27):

$$
\begin{gathered}
q_{11, k}=y_{k-1}^{2}+(k-1)^{2} q_{11, k-1} ; q_{12, k}=y_{k-2} y_{k-1}+(k-1)^{2} q_{12, k-1}, \\
q_{21, k}=y_{k-1} y_{k-2}+(k-1)^{2} q_{21, k-1} ; \quad q_{22, k}=y_{k-2}^{2}+(k-1)^{2} q_{22, k-1} .
\end{gathered}
$$

The equation (25) can be divided into two components as in (28):

$$
\left(\frac{1}{k^{2}}\right)\left(\left[\begin{array}{lr}
y_{k-1}^{2} & y_{k-1} y_{k-2} \\
y_{k-1} y_{k-2} & y_{k-2}^{2}
\end{array}\right]+(k-1)^{2}\left[\begin{array}{ll}
\bar{q}_{11, k-1} & \bar{q}_{12, k-1} \\
\bar{q}_{21, k-1} & \bar{q}_{22, k-1}
\end{array}\right]\right)
$$

The estimated parameter using the second moment expressed in (18), (21) and (26) is presented in (29):

$$
\bar{a}_{k}=\left[\begin{array}{ll}
\bar{a}_{1, k} & \bar{a}_{2, k}
\end{array}\right]=\left[\begin{array}{ll}
\bar{p}_{1, k} & \bar{p}_{2, k}
\end{array}\right]\left[\begin{array}{ll}
\bar{q}_{11, k} & \bar{q}_{12, k} \\
\bar{q}_{21, k} & \bar{q}_{22, k}
\end{array}\right]^{+},
$$

where $\bar{a}_{k}$ and $p_{k}$, are matrices of dimensions: $1 \times 2$, while $q_{k}$ is a matrix of dimension: $2 \times 2$, and is also a pseudoinverse [16]. Then, the simplified stochastic output $\tilde{y}_{k}$ of (13) respect to the estimation parameter of second moment $\bar{a}_{k}$ ends up being (30):

$$
\bar{y}_{k}=\bar{a}_{1} y_{k-1}+\bar{a}_{2} y_{k-2}+\bar{V}_{k} \text {. }
$$

\section{Recursive Stochastic Estimation Algorithm}

From (19), the covariance $p_{k}$ can be obtained as the matrix (31) of $1 \times 2$ being a function of the covariance $q_{k}$.

$$
\begin{gathered}
\left.\left[\begin{array}{ll}
\hat{p}_{1, k} & \hat{p}_{2, k}
\end{array}\right]=\frac{1}{k}\left(\begin{array}{cc}
y_{k} y_{k-1}-V_{k} y_{k-1} & y_{k} y_{k-2}-V_{k} y_{k-2} \\
+(k-1)\left[\bar{q}_{1, k-1} \bar{a}_{1, k-1} \quad \bar{q}_{2, k-1} \bar{a}_{2, k-1}\right.
\end{array}\right]\right) .
\end{gathered}
$$

Using (18) to calculate the estimation parameter, together with (22) and (25) we get (32), then, the stochastic output based on the recursive second moment is (33).

$$
\begin{gathered}
\hat{a}_{k}=\left[\begin{array}{cc}
\hat{a}_{1, k} & \hat{a}_{2, k}
\end{array}\right]=\left[\begin{array}{ll}
\hat{p}_{1, k} & \hat{p}_{2, k}
\end{array}\right]\left[\begin{array}{cc}
\bar{q}_{11, k} & \bar{q}_{12, k} \\
\bar{q}_{21, k} & \bar{q}_{22, k}
\end{array}\right]^{+}, \\
\hat{y}_{k}=\hat{a}_{1} y_{k-1}+\hat{a}_{2} y_{k-2}+\hat{V}_{k} .
\end{gathered}
$$




\section{Functional of Mean Quadratic Error}

The mean quadratic error functional is a measurement to accurately know about the estimation parameter convergence by means of the errors between the normal parameter and the one from the second stochastic moment [18]. Considering the parametric error 1 (34), parametric error 2 (35), error functional 1 (36), and error functional 2 (37):

$$
\begin{gathered}
\bar{e}_{1, k}=a_{1, k}-\bar{a}_{1, k}, \\
\bar{e}_{2, k}=a_{2, k}-\bar{a}_{2, k}, \\
\bar{J}_{1, k}=\left(\frac{1}{k^{2}}\right)\left[\bar{e}_{1, k}^{2}+(\mathrm{k}-1)^{2} \bar{J}_{1, k-1}\right], \\
\bar{J}_{2, k}=\left(\frac{1}{k^{2}}\right)\left[\bar{e}_{2, k}^{2}+(\mathrm{k}-1)^{2} \bar{J}_{2, k-1}\right] .
\end{gathered}
$$

The recursive form has utmost importance, because it generally gives proof of precision between the stochastic estimation parameters and the ones based on the second moment, which are meant to be implemented in a digital system. Considering parametric error 1(38), parametric error 2 (39), error functional 1 (40), error functional 2 (41).

$$
\begin{gathered}
\hat{e}_{1, k}=a_{1, k}-\hat{a}_{1, k}, \\
\hat{e}_{2, k}=a_{2, k}-\hat{a}_{2, k}, \\
\hat{\jmath}_{1, k}=E\left\{\hat{e}_{1, k}^{2}\right\}=\left(\frac{1}{k^{2}}\right)\left[\hat{e}_{1, k}^{2}+(\mathrm{k}-1)^{2} \hat{J}_{1, k-1}\right], \\
\hat{\mathrm{J}}_{2, k}=E\left\{\hat{e}_{2, k}^{2}\right\}=\left(\frac{1}{k^{2}}\right)\left[\hat{e}_{2, k}^{2}+(\mathrm{k}-1)^{2} \hat{J}_{2, k-1}\right] .
\end{gathered}
$$

\section{Experiment}

The stochastic estimation algorithm based on the second moment is meant to be placed as an example.

For the stochastic process the considered parameters are $a 1=0.1+i 0.05 i ; a 2=-$ $0.1+0.05 ; b=0.002 ; c=1 ; d=0.003$.

The identification of the system variable is presented by means of the Fig. 2 and Fig. 3. Where the first fifteen samples of the output responses are matched and hence, tend to be confused with each other. For the recursive case, the identification of the output variables is achieved more quickly. The graphs of the experiment were carried out using Matlab [19, 20].

For a second stage the initial conditions of the recursive stochastic estimation algorithm, for (26) and (27) are: $\operatorname{pr} 1(2)=0.003 ; \operatorname{pr} 2(2)=0.001 ; \operatorname{anr} 1(2)=0.05 ; \operatorname{anr} 2(2)=0.05$. Implementing this values, the result for the parameter estimation is seen in Fig. 4. 
Lumped Parameter Estimation of a Stochastic Process of Second Order ...

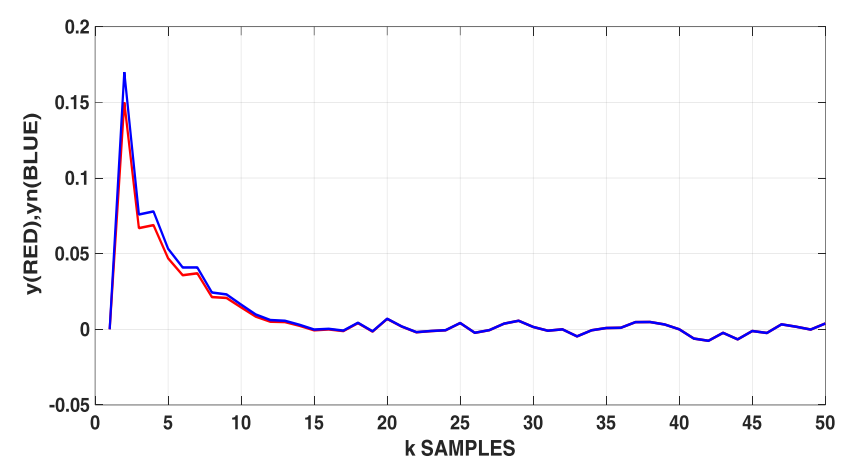

Fig. 2. Stochastic simplified output variable identification.

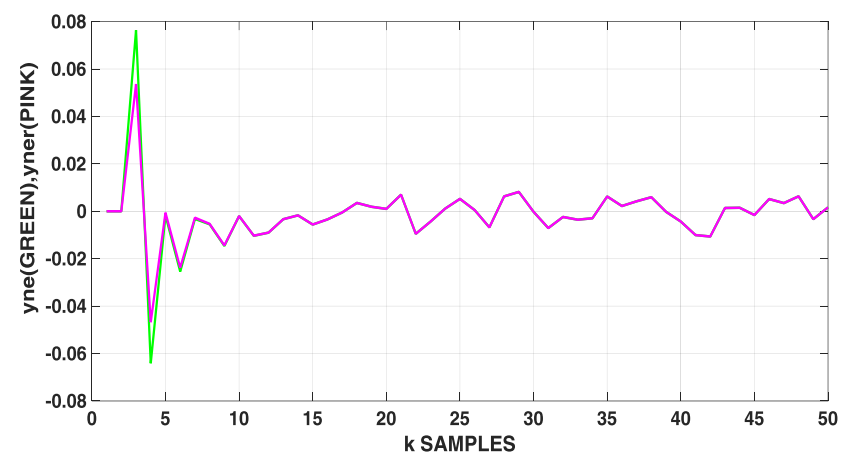

Fig. 3. Stochastic recursive outputs variable identification.

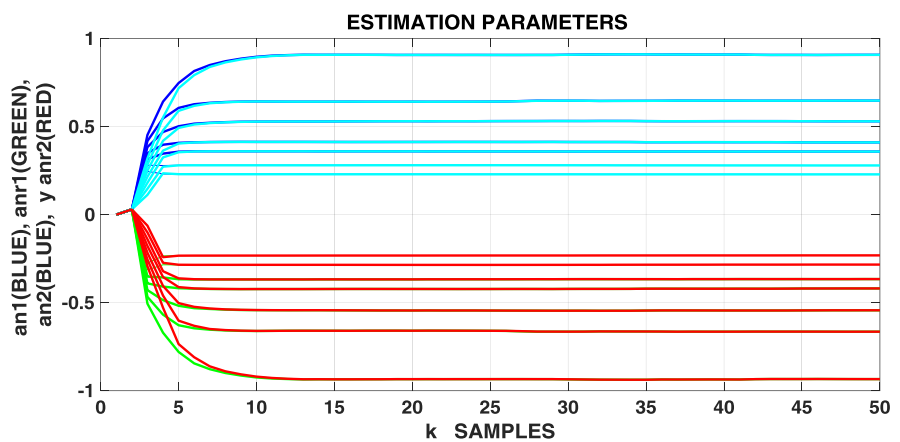

Fig. 4. Estimated stochastic parameters based on the second moment $\bar{a}_{1}$ and $\bar{a}_{2}$ from Eq. (29) and recursive stochastic calculated parameters $\hat{a}_{1}$ and $\hat{a}_{2}$ from Eq. (32).

To have a better idea of the convergence we use the error functional to measure the convergence level for both estimated parameters. The initial conditions for the mean quadratic error functional based on the second moment and the recursiveness, Eqs. (30) and (31) together with (34) and (35), respectively are the following: second mo- 
ment: $\mathrm{J} 1(2)=0.0004 ; \mathrm{J} 2(2)=0.0004$ and recursiveness: $\mathrm{Jr} 1(2)=0.0004 ; \mathrm{Jr} 2(2)=0.0004$. Results are shown in Fig. 5 and Fig. 6.

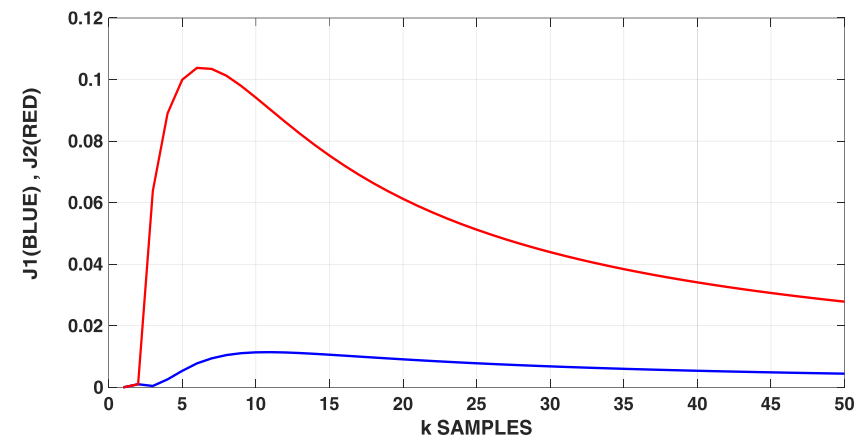

Fig. 5. Graph 6. Error functional of second moment $\overline{J_{1}}$ in blue colour and $\overline{J_{2}}$ in red colour. Remark: Here we deal with a Super Martingale.

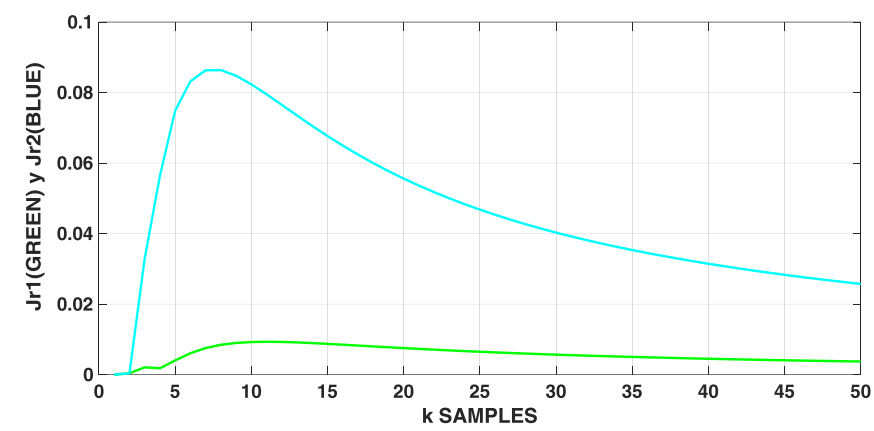

Fig. 6. Graph 7. Error functional of second moment $\overline{\hat{J}}_{1}$ in green colour and $\overline{\hat{J}}_{2}$ in sky-blue colour. Remark: Here we deal again with a Super Martingale.

\section{Conclusions}

The main objective of making the convergence of the two estimation parameters of states $\bar{a}_{k}$, based on the estimation algorithm using the second moment, into the value lost by the parameters of the linearly modelled stochastic states was fulfilled.

The found estimation parameters for the second moment were only able to locate the convergence, when they were subjected to a sign inversion, just as it is described in (1). The convergence range of the estimation parameters did work only within $0<$ $\bar{a}_{k}<1$. The paths of the estimation parameters were always of the Super Martingale form.

The fireproof took place, when the estimation parameters of this proposed algorithm were replaced at the output variable and it was noticed that the experimentally measurable random data was perfectly compared with the a priori data. 
Due to the previous acknowledgements, we come up with the conclusion that the estimation algorithm based on the second moment applied to a random experimental model and represented by a linear stochastic second order model worked out properly.

Also, it was possible to design and simulated experiment with a recursive estimator model $\hat{a}_{k}$, starting with the estimator based on the second moment, whose objective in the future is to be placed at the disposal of whomever wants to implement it on a digital system by only having experimental data of random systems.

Acknowledgments. The authors would like to thank the Instituto Politécnico Nacional, through projects SIP20171418, SIP20170227, and CONACYT, for the support given to carry out this project.

\section{References}

1. Abonyi, J.: Fuzzy Model Identification for Control. Editorial Birhausser, pp. 1-21 (2003)

2. Rivals, I., Personnaz, L.: Black Box Modeling with State Space Neural Networks. (1995)

3. Sinha, A:. Optimal and Robust Control. CRC Press, pp. 179-228 (2007)

4. Lewis, F. L., Xie, L., Popa, D.: Optimal Estimation, Optimal and Robust Estimation. CRC Press, pp. 3-50 (2008)

5. Medel, J., García, C.V.: Estimación de Parámetros Usando la deconvolución y la Pseudoinversa:Descripción e Implementación Recursiva. Revista de Física 56 (1) 54-60 (2009)

6. Aguado, A., Martínez, M.: Identificación y Control Adaptivo. Cap 2. Prentice Hall (2003)

7. Schiavi, E., Muñoz, A. I.: Métodos en Diferencias Finitas para para la Resolución de Problemas de Contorno. (2007)

8. Rivero, R.: Identificacion de Sistemas de Segundo Orden.

9. Elliot, R. J., Aggoun, L., Moore, J. B.: Hidden Markov Models. Estimation and Control. p. 19, Springer (2008)

10. Alavez, J.: Estimación de Parámetros en Ecuaciones Diferenciales Ordinarias. Cap. 1, pp. $1-34$.

11. Heij, C., Ran A., van Schagen, F.: Introduction to Mathematical System Theory. In: Linear Systems, Identification and Control, p. 75. Birkhauser Verlag (2007)

12. Hoel, P. G., Port, S. C., Stone, C. J.: Introduction to Probability Theory. Chapter 4, pp. 92-95. Houghton Mifflin Company (1971)

13. Domínguez, J. A., González-Farías, G., Rodríguez-Dagnino, R. M.: Un procedimiento práctico para estimar el parámetro de forma de la distribución Gaussiana Generalizada. p. 6, Centro de Investigación de Matemáticas.

14. Wilfredo Dominguez C. Convergencia Aleatoria en Espacios de Hilbert. PESQUIMAT. Revista de la F.C.M. de la Universidad mayor de San Marcos. Vol. II, No. 1, pp. 69-70. Lima-Perú (Agosto 1999)

15. Debnath, L., Mikusinski, P.: Hilbert Spaces with Applications. Chap. 3, pp 93-135. Elsevier Academy Press (2005)

16. Medel Juárez, J.J., Zagaceta Álvarez, M.T.: Estimación-Identificación como Filtro Digital Integrado: Descripción e Implementación Recursiva. Revista Mexicana de Física 56 (1) 1-8 (Feb. 2010)

17. Rengifo, C. F.: Una Herramienta para Identificación Recursiva de Sistemas Dinámicos. pp. 1-12 (2004) 
Romeo Urbieta Parrazales, José de Jesús Medel Juárez, Karen Alicia Aguilar Cruz, et al.

18. Santo Orcero, D.: La Matriz Fundamental y la Matriz esencial. Conceptos y aplicaciones. (2002)

19. Lewis, F.L., Xie, L., Popa, D.: Optimal Estimation. In: Optimal and Robust Estimation. Chapter 9, pp. 423-442. CRC Press (2008)

20. Rodríguez del Río, R.: Gráficas con MatLab. Madrid, España.

21. Dukkipatti, R.: Analysis and Design of Control Systems Using Matlab. New Age International (2006)

22. Lagunas, M.A.: Procesos Estocásticos y Estimación de Parámetros. Cap. II (2007)

23. Stroock, D.W.: Probability Theory: An Analytic View. Chapter 4. Cambridge University Press (1994) 